TRANSACTIONS OF THE

AMERICAN MATHEMATICAL SOCIETY

Volume 345, Number 2, October 1994

\title{
MEASURABLE QUOTIENTS OF UNIPOTENT TRANSLATIONS ON HOMOGENEOUS SPACES
}

\author{
DAVE WITTE
}

\begin{abstract}
Let $U$ be a nilpotent, unipotent subgroup of a Lie group $G$, and let $\Gamma$ be a closed subgroup of $G$. Marina Ratner showed that every ergodic $U$-invariant probability measure on the homogeneous space $\Pi G$ is of a simple algebraic form. We use this fundamental new result to show that every measurable quotient of the $U$-action on $\Gamma \backslash G$ is of a simple algebraic form. Roughly speaking, any quotient is a double-coset space $\Lambda \backslash G / K$.
\end{abstract}

\section{INTRODUCTION}

Let $U$ be a Lie subgroup of a Lie group $G$. (All Lie groups in this paper are assumed to be real, finite-dimensional, and second countable.) Any closed subgroup of $G$ can be used to construct an action of $U$, because $U$ acts by translations on any homogeneous space of $G$. In other words, if $\Gamma$ is a closed subgroup of $G$, then there is an action of $U$ on $\Gamma \mid G$ defined by the map $\Gamma \backslash G \times U \rightarrow \Gamma \backslash G:(\Gamma g, u) \mapsto \Gamma g u$. These natural actions have been studied by many authors, because "a large number of interesting examples of dynamical systems can be realized in this fashion ... and because of the ease with which computations can be made in this algebraic setting... " [3, p. 571].

Definition. Recall that a square matrix $A$ is unipotent if it has no eigenvalue other than 1 (in other words, if $A-I$ is nilpotent, where $I$ is the identity matrix). A subgroup $U$ of a Lie group $G$ is unipotent if $\operatorname{Ad}_{G} u$ is a unipotent linear transformation on the Lie algebra of $G$, for every $u \in U$.

The actions of unipotent subgroups have very striking rigidity properties. This is a short way of saying that dynamical properties of such an action are directly related to the algebraic structure of the underlying homogeneous space. For example, work of W. Parry [12], M. Ratner [15], and D. Witte [25] shows that if two such actions are measurably isomorphic (and if $\Gamma \backslash G$ has finite volume), then it is obvious from the algebraic setting that the actions are isomorphic. More recently, M. Ratner has shown that all ergodic invariant measures for such actions can be constructed by purely algebraic methods (see Theorem 1.2 below). In this paper, we use Ratner's result to show that all quotients of these actions can be constructed by purely algebraic methods. (Dynamicists usually refer to quotients as "factors," but this is not consistent with the category-theoretic terminology used throughout mathematics.)

Received by the editors August 16, 1990 and, in revised form, August 31, 1992.

1991 Mathematics Subject Classification. Primary 28C10, 58F11; Secondary 22D40, 28D10, 28D15, 53C30.

(C)1994 American Mathematical Society 
Actions on double-coset spaces $\Lambda \backslash G / K$ provide obvious examples of quotients of the $U$-action on $\Gamma \backslash G$. Roughly speaking, we show that every quotient is one of these obvious examples. Theorem 1.1 provides the precise statement of this result; it requires a few preliminary definitions. If $G$ is abelian, the result reduces to the well-known fact that every quotient of an ergodic toral flow is another toral flow. If $G$ is nilpotent, the result is a theorem of W. Parry [13, Theorem 5.1]; if $G=\mathrm{SL}_{2}(\mathbf{R})$, the result is a theorem of $\mathrm{M}$. Ratner [16] (see also [17, Part II]). The general result is in the spirit of del Junco and Rudolph's [5] classification of the quotients of ergodic actions whose self-joinings are graphs.

Definition. Suppose a group $U$ acts on a measure space $S$. An action of $U$ on a measure space $T$, together with a measure-preserving, $U$-equivariant map from a conull subset of $S$ onto a conull subset of $T$, is a (measurable) quotient of the action on $S$.

Definition. Suppose $\Lambda$ is a closed subgroup of a Lie group $G$, and let $N$ be the largest normal subgroup of $G$ contained in $\Lambda$. For any $a \in G$ and any automorphism $\sigma$ of $G / N$ with $(\Lambda / N)^{\sigma}=\Lambda / N$, there is a corresponding (invertible) affine map:

$$
\Lambda \backslash G \rightarrow \Lambda \backslash G: \Lambda g \mapsto \Lambda g^{\sigma} a .
$$

We use $\operatorname{Aff}(\Lambda \mid G)$ to denote the group of all affine maps on $\Lambda \backslash G$.

Definition. Let $U$ be a subgroup of a Lie group $G$, and let $\Gamma$ be a closed subgroup of $G$. Suppose

(1) $\Lambda$ is a closed subgroup of $G$ containing $\Gamma$; and

(2) $K$ is a closed subgroup of $A f f(\Lambda \backslash G)$ such that

(a) after discarding a set of measure 0 , the orbit space $\Lambda \backslash G / K$ is countably separated; and

(b) $U$ normalizes $K$.

Then the natural $U$-action on $\Lambda \backslash G / K$ is a quotient of the $U$-action on $\Gamma \backslash G$.

Any quotient of the form $\Lambda \mid G / K$, with $\Lambda$ and $K$ as in (1) and (2) is a double-coset quotient of the $U$-action on $\Gamma \backslash G$. A double-coset quotient $\Lambda \backslash G / K$ is central if every affine map in $K$ commutes with every element of $U$.

Remark. Glimm's Theorem [26, Theorem 2.1.14, p. 12] shows that condition (2a) holds if every $K$-orbit on $\Lambda \backslash G$ is locally closed.

Main Theorem 1.1 ("Classification of Quotients"). Suppose $\Gamma \mid G$ is a finitevolume homogeneous space of a Lie group $G$, and $U$ is a nilpotent, unipotent subgroup of $G$ that acts ergodically on $\Gamma \backslash G$. Then every quotient of the $U$-action on $\Pi \backslash G$ is isomorphic to a central double-coset quotient.

The proof of this theorem is based on Ratner's Theorem 1.2 below.

Definition. Let $G$ act on a Borel space $S$. A probability measure $\mu$ on $S$ is homogeneous for a subgroup of $G$ if there is a closed subgroup $X$ of $G$, preserving $\mu$, such that $\mu$ is supported on a single $X$-orbit. Note that $\mu$ is $X$-invariant, but need not be $G$-invariant.

Remark. To show that $\mu$ is homogeneous, it suffices to find a subgroup $X$ of $G$, preserving $\mu$, such that $\mu$ is supported on a single $X$-orbit - there is 
no need to show that $X$ is closed. This is because $\operatorname{Stab}_{G}(\mu)$ is closed and, by definition, preserves $\mu$. (We use $\operatorname{Stab}_{G}(\mu)$ to denote the subgroup consisting of all the elements of $G$ that preserve $\mu$.) Since $X \subset \operatorname{Stab}_{G}(\mu)$, the $X$ orbit supporting $\mu$ is contained in an orbit of $\operatorname{Stab}_{G}(\mu)$; this $\operatorname{Stab}_{G}(\mu)$-orbit supports $\mu$.

Theorem 1.2 (M. Ratner "Classification of Invariant Measures" [19], [20], [21]). Suppose $\Gamma$ is a closed subgroup of a Lie group $G$, and $U$ is a nilpotent, unipotent subgroup of $G$. Then every ergodic $U$-invariant probability measure on $\Gamma \backslash G$ is homogeneous for a subgroup of $G$.

Remark. Ratner originally reported this result with the additional hypotheses that $G$ is connected and that $\Gamma$ is discrete. In $\S 3$, we show that these hypotheses can be eliminated; however, the published version of Ratner's paper does not assume the connectivity of $G$.

Proof of Theorem 1.1. The diagonal embedding of $U$ is a nilpotent, unipotent subgroup of $G \times G$, so Ratner's Theorem 1.2 implies that every ergodic invariant measure for the diagonal $U$-action on $(\Gamma \backslash G) \times(\Gamma \backslash G)$ is homogeneous for a subgroup of $G \times G$. Then Theorem 2.1 below implies that every quotient of the $U$-action on $\Gamma \backslash G$ is isomorphic to a central double-coset quotient, as desired.

Theorem 2.1' . Let $U$ be a subgroup of a Lie group $G$, and let $\Gamma \backslash G$ be a finitevolume homogeneous space of $G$. Assume that the $U$-action on $\Pi G$ is ergodic, and that every ergodic invariant probability measure for the diagonal $U$-action on $(\Gamma \backslash G) \times(\Gamma \backslash G)$ is homogeneous for a subgroup of $G \times G$. Then every quotient of the $U$-action on $\Gamma \mid G$ is isomorphic to a central double-coset quotient.

By passing to a subgroup of $G$, we can apply the classification of quotients even when $\Gamma \backslash G$ does not have finite volume.

Corollary 1.3. Suppose $\Gamma$ is a closed subgroup of a Lie group $G$, and $U$ is a nilpotent, unipotent subgroup of $G$ that acts ergodically on $\Pi G$, with respect to some probability measure $\mu$. Then every quotient of the $U$-action on $(\Gamma \backslash G, \mu)$ is isomorphic to a central double-coset quotient of the $U$-action on a finite-volume homogeneous space of some closed subgroup of $G$.

Proof. Ratner's Theorem 1.2 asserts that $\mu$ is homogeneous, which means there is a closed subgroup $X$ of $G$, preserving $\mu$, such that some $X$-orbit is a conull set. By discarding the complement of this $X$-orbit, we see that the $U$-action on $\Pi \backslash G$ is isomorphic to the $U$-action on a finite-volume homogeneous space $\Lambda \backslash X$ of $X$. Then Theorem 1.1 asserts that every quotient is isomorphic to a central double-coset quotient of the $U$-action on $\Lambda \backslash X$.

If $G$ is connected and semisimple, we can improve the classification of quotients by providing more precise information on the form of the double-coset quotient. There is no need to assume $U$ is nilpotent, because every unipotent subgroup of a connected Lie group is nilpotent (cf. Engel's Theorem [6, $\S$ V.2, pp. 63-67]).

Definition. We say that a homogeneous space $\Gamma \backslash G$ of a Lie group $G$ is faithful if $G$ acts faithfully on $\Gamma \backslash G$ or, equivalently, if $\Gamma$ contains no nontrivial normal subgroup of $G$. 
Corollary 5.1'. Suppose $G$ is a connected, semisimple Lie group, and $\Gamma \backslash G$ is a faithful, finite-volume homogeneous space of $G$. Suppose $U$ is a unipotent subgroup of $G$ that acts ergodically on $\Gamma \backslash G$. Then any quotient of the $U$-action on $\Pi G$ is of the form $\left(N \Gamma^{\prime}\right) \backslash G / K$, where

(1a) $N$ is a closed, normal subgroup of $G$ such that $N \Gamma$ is closed;

(1b) $\Gamma^{\prime}$ is a lattice containing $\Gamma$; and

(2) $K$ is a compact group, consisting of affine maps on $\left(N \Gamma^{\prime}\right) \backslash G$ that centralize $U$.

The Classification of Invariant Measures and the Classification of Quotients can also be applied to certain natural actions of semisimple groups and their lattice subgroups. For example, the discrete group $\mathrm{SL}_{n}(\mathrm{Z})$ acts by affine maps on the torus $\mathbb{T}^{n}$. (Assume $n \geq 2$, so that the action is ergodic.) The Classification of Invariant Measures implies that the Haar measure on $\mathbf{T}^{n}$ is the only nonatomic, ergodic invariant probability measure for this action (see the remark following Corollary $5.8^{\prime}$, and note that $\mathbb{T}^{n}$ has no nontrivial, proper, connected, $\mathrm{SL}_{n}(\mathbb{Z})$-invariant subgroups). The ergodic measures for the diagonal action on $\mathbf{T}^{n} \times \mathbb{T}^{n}$ can also be classified, which leads to the conclusion that any nontrivial quotient of the action on $T^{n}$ is the action on an $n$-torus covered by $T^{n}$ or on the orbifold constructed from a certain $(\mathbb{Z} / 2 Z)$-action on such an $n$-torus (see Example 5.9). For the case $n=2$, these results are due to $K$. Park [11].

Definition. Let $G$ be a connected, semisimple Lie group that acts by automorphisms on some connected Lie group $N$. If $\Gamma$ is a lattice in $G$, and $\Lambda$ is any $\Gamma$-invariant, closed subgroup of $N$, then the action of $\Gamma$ on $N$ factors through to an action on $\Lambda \backslash N$. In this situation, let us say that $\Gamma$ acts automorphically on $\Lambda \backslash N$.

If $\Gamma$ acts automorphically on $\Lambda \backslash N$, then we can form the semidirect product group $\Gamma \propto N$, and this group acts by affine maps on $\Lambda \backslash N$.

Corollary 5.8'. Let $\Gamma$ be a lattice in a connected, semisimple Lie group $G$ with no compact factors. Suppose $G$ acts by automorphisms on some connected Lie group $N$. If $\Lambda$ is any $\Gamma$-invariant, closed subgroup of $N$, then every ergodic $\Gamma$-invariant probability measure on $\Lambda \backslash N$ is homogeneous for a subgroup of the semidirect product $\Gamma \times N$.

The conclusion of the corollary can also be stated as follows. There is an element $s$ of $\Lambda \backslash N$ and a connected, closed, $\Gamma$-invariant subgroup $M$ of $N$ such that the $\Gamma$-orbit of $s$ is finite, and the $M$-orbit of $s$ supports an $M$ invariant probability measure. Since $M$ is $\Gamma$-invariant, then the $M$-orbit of each point in the $\Gamma$-orbit of $s$ supports an $M$-invariant probability measure. The measure $\mu$ is the (normalized) sum of these finitely many $M$-invariant measures.

Corollary 1.4. Let $\Gamma$ be a lattice in a connected, semisimple Lie group $G$ with no compact factors. Suppose $G$ acts by automorphisms on some connected Lie group $N$, and suppose the $\Gamma$-action on $\Lambda \backslash N$ is ergodic, where $\Lambda \backslash N$ is a finitevolume homogeneous space of $N$ such that $\Lambda$ is $\Gamma$-invariant. Then every quotient of the $\Gamma$-action is a central double-coset quotient. 
The conclusion of the corollary means that every quotient is of the form $\Lambda^{\prime} \backslash N / K$, where

(1) $\Lambda^{\prime}$ is a closed, $\Gamma$-invariant subgroup of $N$ containing $\Lambda$; and

(2) $K$ is a closed group of affine maps on $\Lambda^{\prime} \backslash N$ such that

(a) after discarding a set of measure 0 , the orbit space $\Lambda^{\prime} \backslash N / K$ is countably separated; and

(b) every affine map in $K$ commutes with every element of $\Gamma$.

Proof. Corollary 5.8 implies that every ergodic $\Gamma$-invariant measure for the diagonal $\Gamma$-action on $(\Lambda \backslash N) \times(\Lambda \backslash N)$ is homogeneous for a subgroup of $\Gamma \times$ $(N \times N)$. Therefore, Theorem 2.1 asserts that every quotient is a central doublecoset quotient.

If $G$ is a connected, simple Lie group with finite center and $R-\operatorname{rank}(G) \geq$ 2 then, modulo a null set, all the known volume-preserving, ergodic actions of $G$ on a compact manifold $M$ can be constructed by embedding $G$ in some connected Lie group $H$ and forming a central double-coset quotient $\Lambda \backslash H / K$. It is natural to try to obtain new examples by taking quotients of these known actions, but the Classification of Quotients shows that no new actions will be produced.

Corollary 5.6'. Let $G$ be a connected, semisimple Lie group with no compact factors. Immerse $G$ in some connected Lie group $H$, and let $\Lambda \backslash H$ be a finitevolume homogeneous space of $H$, on which $G$ acts ergodically. Then every measurable quotient of the $G$-action on $\Lambda \backslash H$ is a central double-coset quotient.

Acknowledgment. I am indebted to Marina Ratner, Cesar Silva, and an anonymous referee, for comments that corrected and clarified the results of this paper.

\section{QUOTIENTS OF UNIPOTENT TRANSLATIONS}

Theorem 2.1. Let $U$ be a subgroup of a Lie group $G$, and let $\Gamma \backslash G$ be a finitevolume homogeneous space of $G$. Assume that the $U$-action on $\Pi \backslash G$ is ergodic, and that every ergodic invariant probability measure for the diagonal $U$-action on $(\Pi G) \times(\Pi \backslash G)$ is homogeneous for a subgroup of $G \times G$. Then every quotient of the U-action on $\Gamma \backslash G$ is isomorphic to a central double-coset quotient.

Proof. Replacing $U$ by its closure, we may assume $U$ is a closed subgroup of $G$. Let $\psi:(\Gamma \backslash G, \mu) \rightarrow(T, \nu)$ be a measure-preserving, $U$-equivariant, Borel map, where $\mu$ is the $G$-invariant probability measure on $\Gamma \backslash G$. Let

$$
M=\left\{(s, t) \in \frac{G}{\Gamma} \times \frac{G}{\Gamma} \mid \psi(s)=\psi(t)\right\} .
$$

To study the quotient $(T, \nu)$, we construct a certain $U$-invariant measure $\rho$ supported on $M$ (cf. the proof of [17, Corollary 5, p. 301]). (This measure is known as the relatively independent joining.) Since $\psi$ is measure preserving, we can decompose $\mu$ essentially uniquely into probability measures $\mu_{t}$ supported on the fibers $\psi^{-1}(t)$ of $\psi[22, \S 3$, pp. 26-33]. This means that, for any Borel set $A \subset \Gamma \backslash G$, we have

$$
\mu(A)=\int_{T} \mu_{t}(A) d \nu(t) .
$$


The desired probability measure $\rho$ on $(\Gamma \backslash G) \times(\Gamma \backslash G)$ is defined by

$$
\rho(A \times B)=\int_{T} \mu_{t}(A) \mu_{t}(B) d \nu(t),
$$

for any Borel sets $A, B \subset \Gamma \backslash G$. It is clear that $\rho$ is supported on $M$. It is not hard to see that $\rho$ is $U$-invariant, and projects to $\mu$ on both the first and second factor of $(\Gamma \backslash G) \times(\Gamma \backslash G)$. Since $U$ is ergodic on $\Pi \backslash G$, this implies that each ergodic component of $\rho$ projects to $\mu$ on both factors. Let

$$
\Lambda=\{\lambda \in G \mid \psi(\Gamma \lambda s)=\psi(\Gamma s) \text { for a.e. } s \in G\} .
$$

Then $\Lambda$ is a closed subgroup of $G$ (see Lemma 2.3). By changing the quotient $(S, \nu)$ on a null set, we may assume $\psi(\Gamma \lambda s)=\psi(\Gamma s)$ for every $s \in G$ and $\lambda \epsilon$ $\Lambda$ [26, Proposition B.5, pp. 198-199]. This means that the map $\bar{\psi}: \Lambda \backslash G \rightarrow T$ defined by $\bar{\psi}(\Lambda g)=\psi(\Gamma g)$ is well defined. Let

$$
L=\left\{\varphi \in C_{\mathrm{Aff}(\Lambda \backslash G)}(U) \mid \bar{\psi}(\varphi(s))=\bar{\psi}(s) \text { for a.e. } s \in \frac{G}{\Lambda}\right\},
$$

where $C_{\mathrm{Aff}(\Lambda \backslash G)}(U)$ is the group of invertible affine maps on $\Lambda \backslash G$ that centralize $U$. Note that $L$ is closed (see Lemma 2.2).

It will suffice to show, for almost all $(\Gamma s, \Gamma t) \in M$ (w.r.t. $\rho$ ), that there is some $\varphi \in L$ with $\varphi(\Lambda s)=\Lambda t$, because this implies that, modulo a null set, the original quotient $(T, \nu)$ is isomorphic to the quotient $\Lambda \backslash G / L$.

Step 1. For a.e. $(\Gamma s, \Gamma t) \in M$, there is a closed subgroup $X$ of $G \times G$ such that (1) for almost every $x \in X$, we have $(\Gamma s, \Gamma t) \cdot x \in M$, (2) the subgroup $X$ projects surjectively onto each of the two factors of $G \times G$, and (3) the $X$-orbit $(\Gamma s, \Gamma t) \cdot X$ is $U$-invariant. Being an ergodic $U$-invariant measure on $(\Gamma \mid G)^{2}$, the ergodic component of $\mu$ containing $(s, t)$ must be the homogeneous measure on an orbit of some closed subgroup $X$ of $G \times G$. Each ergodic component is supported on $M$, so (1) almost every point of the orbit $(\Gamma s, \Gamma t) \cdot X$ is in $M$. We know that the ergodic component projects to the $G$-invariant measure $\mu$ on both the first and second factor of $(\Pi \backslash G)^{2}$, so (2) $X$ must project surjectively onto both factors of $G^{2}$. Since ergodic components, by definition, are $U$-invariant, the homogeneous $X$-invariant measure on $(\Gamma s, \Gamma t) \cdot X$ is $U$ invariant. It is immediate that (3) $(\Gamma s, \Gamma t) \cdot X$ is $U$-invariant.

Step 2. The orbit $(\Lambda s, \Lambda t) \cdot X$ is the graph of a well-defined affine map $\varphi: \Lambda \backslash G \rightarrow$ $\Lambda \backslash G$. Because $X$ projects onto both factors of $G \times G$, it will suffice to show $X \cap\left(s^{-1} \Lambda s \times G\right) \subset s^{-1} \Lambda s \times t^{-1} \Lambda t$. (Then, by symmetry, the inverse of $\varphi$ is also well-defined.) For simplicity, assume $\Gamma s=\Gamma t=\Gamma$, so that we wish to show $X \cap(\Lambda \times G) \subset \Lambda \times \Lambda$. Let $(\lambda, h) \in X \cap(\Lambda \times G)$. Given any $g \in G$, there is some $g^{\prime} \in G$ with $\left(g^{\prime}, g\right) \in X$. Then (assuming $g$ misses some exceptional set of measure 0$)$ we have $\psi(\Gamma g)=\psi\left(\Gamma g^{\prime}\right)$ because $(\Gamma, \Gamma) \cdot X=(\Gamma s, \Gamma t) \cdot X$ is essentially contained in $M$. Because $\lambda \in \Lambda$, we have $\psi\left(\Gamma g^{\prime}\right)=\psi\left(\Gamma \lambda g^{\prime}\right)$. Since both $(\lambda, h)$ and $\left(g^{\prime}, g\right)$ belong to the subgroup $X$, we have $\left(\lambda g^{\prime}, h g\right) \in X$. Therefore (again assuming $g$ misses some exceptional set of measure 0 ) we have $\psi\left(\Gamma \lambda g^{\prime}\right)=\psi(\Gamma h g)$. In summary, we have

$$
\psi(\Gamma g)=\psi\left(\Gamma g^{\prime}\right)=\psi\left(\Gamma \lambda g^{\prime}\right)=\psi(\Gamma h g)
$$

for a.e. $g \in G$. Hence, by the definition of $\Lambda$, we have $h \in \Lambda$ as desired. 
Step 3. $\varphi$ belongs to $L$. (1) Because the orbit $(\Gamma s, \Gamma t) \cdot X$ is $U$-invariant, it is immediate that $(\Lambda s, \Lambda t) \cdot X$ is $U$-invariant in $(\Lambda \mid G)^{2}$. This means the graph of $\varphi$ is $U$-invariant, so $\varphi$ centralizes $U$. (2) Because the orbit $(\Gamma s, \Gamma t) \cdot X$ is (essentially) contained in $M$, we know that $\bar{\psi}(\varphi(p))=\bar{\psi}(p)$ for almost all $p \in \Lambda \backslash G$.

Lemma 2.2. Suppose a Lie group $G$ acts on a Borel space $S$. For any Borel function $\psi$ on $S$, let

$$
L=\{g \in G \mid \psi(s g)=\psi(s) \text { for a.e. } s \in S\} .
$$

Then $L$ is a closed subgroup of $G$.

Proof. Let $T$ be the image space of $\psi$, which simply means $\psi: S \rightarrow T$. As described in [26, pp. 49-50], let $F(S, T)$ be the space of measurable functions $S \rightarrow T$, two functions being identified if they agree almost everywhere. The topology of convergence in measure makes $F(S, T)$ a metrizable space, and $G$ acts continuously on $F(S, T)$. Hence, the stabilizer of any point in $F(S, T)$ is a closed subgroup of $G$. In particular, we see that $L$, the stabilizer of $\psi$, is a closed subgroup.

Lemma 2.3. Let $\Gamma$ be a closed subgroup of a Lie group $G$. Suppose $\psi$ is a measurable function on $\Gamma \mid G$, and let

$$
\Lambda=\{\lambda \in G \mid \psi(\Gamma \lambda s)=\psi(\Gamma s) \text { for a.e. } s \in G\} .
$$

Then $\Lambda$ is a closed subgroup of $G$.

Proof. Let $T$ be the image space of $\psi$, which simply means $\psi: \Gamma \backslash G \rightarrow T$. Letting $S=G$, we can lift $\psi$ to a function $\tilde{\psi}: S \rightarrow T$ defined by $\tilde{\psi}(S)=$ $\psi(\Gamma s)$. Then

$$
\Lambda=\{g \in G \mid \tilde{\psi}(g s)=\tilde{\psi}(s) \text { for a.e. } s \in S\} .
$$

There is an action of $G$ on $S$ defined by $s \cdot g=g^{-1} s$ for $s \in S$ and $g \in G$. For this action, and with $\tilde{\psi}$ in the place of $\psi$, the subgroup $L$ of Lemma 2.2 is $\Lambda$, so $\Lambda$ is closed.

\section{NONDISCRETE SUBGROUPS AND DISCONNECTED GROUPS}

M. Ratner's original statement of Theorem 1.2 required $\Gamma$ to be discrete and $G$ to be connected. In this section, we show how to eliminate these hypotheses. The published version of Ratner's paper does not require $G$ to be connected, but the alternate approach presented here may be useful for generalizations.

Theorem 1.2' . Suppose $\Gamma$ is a closed subgroup of a Lie group $G$, and $U$ is a nilpotent, unipotent subgroup of $G$. Then every ergodic, $U$-invariant probability measure on $\Gamma \backslash G$ is homogeneous for a subgroup of $G$.

Notation. We use $X^{\circ}$ to denote the identity component of a Lie group $X$.

It is quite easy to eliminate the discreteness assumption on $\Gamma$. The Borel Density Theorem (see Corollary 4.3 below) implies that, replacing $\mu$ by a translate and $U$ by a conjugate, we may assume $U \subset N_{G}\left(\Gamma^{\circ}\right)$ and $\mu$ is supported on $\Gamma \backslash N_{G}\left(\Gamma^{\circ}\right)$. Replacing $G$ by $N_{G}\left(\Gamma^{\circ}\right)$, then we may assume $\Gamma^{\circ} \triangleleft G$. There is no loss of generality in modding out $\Gamma^{\circ}$, so we may assume $\Gamma$ is discrete. 
We now need to eliminate the connectedness assumption on $G$. The idea is simple: we want to embed $G$ as a closed subgroup of some connected Lie group. Unfortunately, some groups (such as $\mathbb{Q}$ ) cannot be embedded as a closed subgroup of any connected Lie group, so the proof involves some shenanigans.

It will be helpful to generalize the notion of homogeneity. For the measures that interest us, Lemma 3.1 shows that the a priori weaker notion of stable homogeneity is equivalent to homogeneity.

Definition. Let $G$ act on a Borel space $S$. A probability measure $\mu$ on $S$ is stably homogeneous for a subgroup of $G$ if there is a closed subgroup $X$ of $G$, such that some $X$-orbit has nonzero measure (w.r.t. $\mu$ ), and such that $X$ preserves the measure obtained by restricting $\mu$ to this $X$-orbit.

Lemma 3.1. Let a Lie group $G$ act on a Borel space $S$ and let $\mu$ be a probability measure on $S$. Assume $\mu$ is ergodic, with respect to some unipotent subgroup $U$ of $G$. Then the following are equivalent:

(a) $\mu$ is homogeneous for a subgroup of $G$;

(b) $\mu$ is stably homogeneous for a subgroup of $G$;

(c) $\mu$ is stably homogeneous for a subgroup of $G^{\circ}$.

Proof. (a) $\Rightarrow$ (b) Obvious.

(b) $\Rightarrow$ (c) There is a closed subgroup $X$ of $G$, such that some $X$-orbit has nonzero measure (w.r.t. $\mu$ ), and such that $X$ preserves the measure obtained by restricting $\mu$ to this $X$-orbit. Since $X / X^{\circ}$ is countable (recall that all Lie groups are assumed to be second countable), this $X$-orbit is the union of countably many $X^{\circ}$-orbits, so some $X^{\circ}$-orbit must have nonzero measure. It is easy to see that $X^{\circ}$ must preserve the restriction of $\mu$ to this orbit, because $X$ preserves the restriction of $\mu$ to the ambient $X$-orbit.

(c) $\Rightarrow$ (a) There is a closed subgroup $X$ of $G^{\circ}$, such that some $X$-orbit $s X$ has nonzero measure (w.r.t. $\mu$ ), and such that $X$ preserves the measure obtained by restricting $\mu$ to this $X$-orbit. From the proof of $(b) \Rightarrow(c)$, we may assume $X$ is connected.

Note that any two $U$-translates of $s X$ either are equal or intersect in a null set. To see this, identify the orbit $s G$ with a coset space $\Gamma \backslash G$ of $G$. Then each $U$-translate of $s X$ is a connected, immersed submanifold. The restriction of $\mu$ to $s X$, being $X$-invariant, is equivalent to Lebesgue measure on this submanifold. By the $U$-invariance of $\mu$, then the restriction of $\mu$ to any $U$ translate of $s X$ is equivalent to Lebesgue measure. If two $U$-translates are not equal, then their intersection is a submanifold of lower dimension, hence their intersection is a null set (with respect to Lebesgue measure and, equivalently, with respect to $\mu$ ).

On the other hand, any two $U$-translates have the same (nonzero) measure and, by ergodicity, the $U$-translates of $s X$ cover a set of full measure. Therefore, there must be only finitely many $U$-translates, and the union of these translates is a conull set. This implies that $U$ normalizes $X$ (see Lemma 4.4). Hence $X$ preserves $\mu$, because $X=u^{-1} X u$ preserves the restriction of $\mu$ to each translate $s X u$. Then $\mu$ is supported on the orbit $s X U$ of the subgroup $X U$, and $X U$ preserves $\mu$.

In a series of steps, we now eliminate the connectedness assumption on $G$. 
Step 1. We may assume $G=G^{\circ} U=\Gamma G^{\circ}$. Because $G^{\circ} \backslash G$ is countable (recall that all Lie groups are assumed to be second countable), we know that $\left(G^{\circ} U\right) \backslash G$ is countable. This means there are only countably many $\left(G^{\circ} U\right)$-orbits in $\Gamma \backslash G$, so some $\left(G^{\circ} U\right)$-orbit must have nonzero measure. (In fact, since $U$ acts ergodically, this $\left(G^{\circ} U\right)$-orbit must be a conull set.) If the restriction of $\mu$ to this $\left(G^{\circ} U\right)$-orbit is homogeneous for a subgroup of $G^{\circ} U$, then $\mu$ is stably homogeneous. Lemma 3.1 then asserts that $\mu$ is homogeneous for a subgroup of $G$. Therefore, we may replace $G$ with $G^{\circ} U$.

Because $G / G^{\circ}$ is countable, some $G^{\circ}$-orbit must have nonzero measure. Since $G=G^{\circ} U$ and $G^{\circ} \triangleleft G$, any two $G^{\circ}$-orbits are $U$-translates of each other, so the $U$-invariance of $\mu$ implies that any two $G^{\circ}$-orbits have the same measure. Since any two distinct $G^{\circ}$-orbits must be disjoint, and $\mu$ is a probability measure, this implies that there are only finitely many $G^{\circ}$-orbits in $\Gamma \backslash G$. This means that the subgroup $\Gamma G^{\circ}$ is of finite index in $G$. Lemma 4.7 shows that we may replace $G$ by any subgroup of finite index, so we may assume $G=\Gamma G^{\circ}$.

Step . 2. Let $\Gamma_{0}=\Gamma \cap G^{\circ}$. The action of $G$ on $\Gamma \backslash G$ by translations is naturally isomorphic to an action of $G$ on $\Gamma_{0} \backslash G^{\circ}$ by affine maps, so there is a natural homomorphism $G \rightarrow \operatorname{Aff}\left(\Gamma_{0} \backslash G^{\circ}\right)$. Since $G=\Gamma G^{\circ}$, the natural inclusion of $\Gamma_{0} \backslash G^{\circ}$ in $\Gamma \backslash G$ is a bijection. This means that the action of $G$ by translations on $\Gamma, G$ is naturally isomorphic to an action of $G$ on $\Gamma_{0} \backslash G^{\circ}$.

We now verify that the action on $\Gamma_{0} \backslash G^{\circ}$ is by affine maps. Given $g \in G$, there is some $\gamma \in \Gamma$ with $\gamma^{-1} g \in G^{\circ}$. For any $s \in G^{\circ}$, we have $\Gamma s g=$ $\Gamma\left(\gamma^{-1} s \gamma\right)\left(\gamma^{-1} g\right)$. This means that the action of $g$ on $\Gamma_{0} \backslash G^{\circ}$ is the affine map whose automorphism component is conjugation by $\gamma$, and whose translation component is $\gamma^{-1} \mathrm{~g}$.

Step 3. We may assume $G^{\circ}$ is simply connected, the natural homomorphism

$$
G^{\circ} \rtimes N_{\mathrm{Aut}\left(G^{\circ}\right)}\left(\Gamma_{0}\right) \rightarrow \operatorname{Aff}\left(\Gamma_{0} \backslash G^{\circ}\right)
$$

is a covering map, and that the natural homomorphism $G \rightarrow \operatorname{Aff}\left(\Gamma_{0} \backslash G^{\circ}\right)$, defined in Step 2, lifts to an injective homomorphism $\rho: G \rightarrow G^{\circ} \rtimes N_{\mathrm{Aut}\left(G^{\circ}\right)}\left(\Gamma_{0}\right)$. Let $N_{0}$ be the largest normal subgroup of $G^{\circ}$ contained in $\Gamma_{0}$. Because $\Gamma_{0}$ is discrete, it is obvious that $N_{0}$ is discrete. Therefore, any automorphism $\varphi$ of $G^{\circ} / N_{0}$ lifts (uniquely) to an automorphism $\tilde{\varphi}$ of the universal cover $\widetilde{G}^{\circ}$ of $G^{\circ}$. Letting $\widetilde{\Gamma_{0}}$ denote the inverse image of $\Gamma_{0}$ in $\widetilde{G^{\circ}}$, we note that $\varphi$ normalizes $\Gamma_{0}$ if and only if $\tilde{\varphi}$ normalizes $\widetilde{\Gamma_{0}}$. Hence, the natural map $N_{\text {Aut }\left(\widetilde{G^{\circ}}\right)}\left(\widetilde{\Gamma_{0}}\right) \rightarrow N_{\text {Aut }\left(G^{\circ} / N_{0}\right)}\left(\Gamma_{0}\right)$ is an isomorphism. Combining this with the covering map $\widetilde{G^{\circ}} \rightarrow G^{\circ} / N_{0}$, we see that the natural map $\widetilde{G^{\circ}} \times N_{\text {Aut }\left(\widetilde{G}^{\circ}\right)}\left(\widetilde{\Gamma_{0}}\right) \rightarrow$ $\operatorname{Aff}\left(\Gamma_{0} \backslash G^{\circ}\right)$ is a covering map.

Each element $g$ of $G$ has an image in $\operatorname{Aff}\left(\Gamma_{0} \backslash G^{\circ}\right)$ under the natural homomorphism $G \rightarrow \operatorname{Aff}\left(\Gamma_{0} \backslash G^{\circ}\right)$, and this image lifts (nonuniquely) to an element of $\widetilde{G^{\circ}} \times N_{\text {Aut }\left(\widetilde{G^{\circ}}\right)}\left(\widetilde{\Gamma_{0}}\right)$. Let $\widetilde{G}$ be the collection of all possible lifts of the images of all possible elements of $G$. Then $\widetilde{G}^{\circ}=\widetilde{G}^{\circ}$ is simply connected. By replacing $G$ and $\Gamma_{0}$ with $\widetilde{G}$ and $\widetilde{\Gamma_{0}}$, we accomplish the desired reduction.

Definition. A real-algebraic group is a Lie group that is a subgroup of finite index in the real points of an (affine) algebraic group defined over $\mathbf{R}$. (The books of 
Humphreys [7, §34], Raghunathan [14, 2 of Preliminaries], and Zimmer [26, \$3.1] contain useful information on algebraic groups over $\mathbf{R}$.)

The theory of algebraic groups provides a notion of unipotence for elements of a real-algebraic group. To avoid hopeless confusion with the definition of unipotence used in this paper, we refer to elements as being algebraically unipotent if they are unipotent as elements of an algebraic group. (In a real-algebraic group, every algebraically unipotent element is unipotent, but the converse fails if $Z(G)$ is not algebraically unipotent. In general, an element of a Lie group $G$ is unipotent iff $\operatorname{Ad}_{G} u$ is an algebraically unipotent element of the real-algebraic group $\mathrm{GL}(\mathscr{G})$, where $\mathscr{G}$ is the Lie algebra of $G$.)

Definition. A Lie group is almost connected if it has only finitely many connected components. For example, every real-algebraic group is almost connected [10, Appendix, pp. 254-255].

Step 4 (The Main Step). We may assume $G$ can be embedded as a closed subgroup of a connected Lie group $Y$, in such a way that $U$ is a unipotent subgroup of $Y$. From Step 3, we have a homomorphism $\rho: G \rightarrow G^{\circ} \rtimes N_{\text {Aut }\left(G^{\circ}\right)}\left(\Gamma_{0}\right)$. By modding out $G^{\circ}$, we obtain a homomorphism

$$
\bar{\rho}: G \rightarrow N_{\text {Aut }\left(G^{\circ}\right)}\left(\Gamma_{0}\right) \subset \operatorname{Aut}\left(G^{\circ}\right) .
$$

Let $U^{*}$ be the Zariski closure of $\bar{\rho}(U)$ in Aut $\left(G^{\circ}\right)$. Then, because $G=G^{\circ} U$, we have $\rho(G) \subset G^{\circ} \rtimes U^{*}$. Note that the group $G^{\circ} \rtimes U^{*}$ is almost connected, and $\rho(U)$ is a unipotent subgroup (because $\left(G^{\circ} \times U^{*}\right) / G^{\circ} \cong U^{*}$ is nilpotent, and each element of $U^{*}$ is Ad-unipotent on $\left.G^{\circ}\right)$. Unfortunately, the image of $G$ may not be closed, so we are not done yet.

Let $\bar{U}$ be the closure of the image of $U$ in $G^{\circ} \times \operatorname{Aut}\left(G^{\circ}\right)$. Note that $\bar{U}$ preserves $\mu$, because $G^{\circ} \times \operatorname{Aut}\left(G^{\circ}\right)$ acts continuously on the space of probability measures $[26, \S 3.2$, p. 38]. Now $\bar{U}$ is a closed, nilpotent subgroup of the almostconnected group $G^{\circ} \rtimes \operatorname{Aut}\left(G^{\circ}\right)$. Replacing $G$ by a subgroup of finite index (see Lemma 4.7 below), we may assume $\bar{U}$ is a subgroup of a connected group. Then Lemma 4.8 below asserts that $\bar{U}$ has a finitely-generated, nilpotent subgroup $X$ that is ergodic.

Let $H=G^{\circ} X<G^{\circ} \rtimes \operatorname{Aut}\left(G^{\circ}\right)$. We know that $H / G^{\circ} \cong X /\left(G^{\circ} \cap X\right)$ is a finitely-generated group isomorphic to a subgroup of $\operatorname{Aut}\left(G^{\circ}\right)$, and Lemma 4.1 asserts that $\operatorname{Aut}\left(G^{\circ}\right)$ is real algebraic. Therefore, $H / G^{\circ}$ has a torsion-free subgroup of finite index [14, Corollary 6.14, p. 95$]$ so, replacing $G$ by a subgroup of finite index (see Lemma 4.7), we may assume $H / G^{\circ}$ is torsion free. Furthermore, $H / G^{\circ}$ is nilpotent because $X$ is nilpotent. Putting all this together, we can apply Lemma 4.6, and conclude that there is a closed embedding $\pi: H / G^{\circ} \rightarrow N$ of $H / G^{\circ}$ in some connected, nilpotent Lie group $N$.

Let $i: H \hookrightarrow G^{\circ} \rtimes U^{*}$ be the natural inclusion. Then the homomorphism

$$
\sigma: H \rightarrow\left(G^{\circ} \times U^{*}\right) \times N \text { defined by } \sigma(h)=(i(h), \pi(h))
$$

embeds $H$ as a closed subgroup of an almost-connected Lie group $Y$. Note that $i(X)$ is a unipotent subgroup of $Y$, because $Y / G^{\circ}$ is nilpotent, and $X$ (being in the closure of $U$ ) is Ad-unipotent on $G^{\circ}$.

Now $H=G^{\circ} X$ acts transitively on $\Gamma \backslash G$, and has a unipotent subgroup (namely $X$ ) that preserves $\mu$ and is ergodic. Suppose $\mu$ is homogeneous for 
a subgroup of $H$. Then Lemma 3.1 asserts that $\mu$ is stably homogeneous for a subgroup of $H^{\circ}=G^{\circ}$. Then Lemma 3.1 implies that $\mu$ is homogeneous for a subgroup of $G$.

Thus, it will suffice to show that $\mu$ is homogeneous for a subgroup of $H$. This means that, by replacing $G$ and $U$ with $H$ and $X$, we may assume $G$ is isomorphic to a closed subgroup of an almost-connected Lie group $Y$, and that $U$ is a unipotent subgroup of $Y$. Passing to a subgroup of finite index (see Lemma 4.7), we may assume $Y$ is connected.

Step 5. We may assume that $G$ is connected. We may assume $G$ is a closed subgroup of some connected Lie group $Y$, and that $U$ is a unipotent subgroup of $Y$. Then $\Gamma \mid G$ is a closed subset of $\Gamma Y$, so $\mu$ is a $U$-invariant probability measure on $\Pi Y$. Assume we know that $\mu$ is homogeneous for a subgroup of $Y$. This means there is a closed subgroup $X$ of $Y$, preserving $\mu$, such that $\mu$ is supported on a single $X$-orbit $s X$. Then $\mu\left(s X^{\circ}\right)>0$, and the restriction of $\mu$ to this $X^{\circ}$-orbit is equivalent to Lebesgue measure on the orbit. Because we know that $\mu$ is supported on $\Gamma \backslash G$, we may assume $s \in \Gamma \backslash G$.

Suppose $X^{\circ}$ is not contained in $G$. Since $\Gamma$ is discrete, then the tangent space to $s X$ at $s$ is not contained in the tangent space to $\Gamma G$ at $s$. This means that the submanifold $(s X) \cap(\Gamma \mid G)$ of $s X$ has positive codimension. Hence this submanifold is a null set (with respect to Lebesgue measure and, equivalently, with respect to $\mu$ ). This is impossible, because $s X$ has positive measure and $\mu$ is supported on $\Gamma \backslash G$.

We now know $X^{\circ} \subset G$. Hence $\mu$ is stably homogeneous for a subgroup of $G$. By Lemma 3.1, then $\mu$ is homogeneous for a subgroup of $G$, as desired. Thus, all that remains is to prove our assumption, namely, that $\mu$ is homogeneous for a subgroup of $Y$. This means that we may replace $G$ with the connected group $Y$.

\section{SOME TECHNICAL LEMMAS}

Lemma 4.1 (see proofs of [26, Proposition 3.1.6 and Theorem 3.2.5(ii)]). Let $G$ be a connected, simply-connected Lie group. Then Aut $(G)$ is (naturally isomorphic to) a real-algebraic group, and the normalizer of any connected subgroup is Zariski closed.

Theorem 4.2 (Dani's version of the Borel Density Theorem [4, Corollary 2.6]). Suppose $\Gamma^{*}$ is an algebraic subgroup of a real-algebraic group $G^{*}$, and let $\nu$ be a probability measure on $\Gamma^{*} \backslash G^{*}$. Set

$$
G_{\nu}^{*}=\left\{g \in G^{*} \mid \text { the } g \text {-action on } \Gamma^{*} \backslash G^{*} \text { preserves } \nu\right\}
$$

and

$$
N_{\nu}^{*}=\left\{g \in G^{*} \mid s g=s \text { for all } s \in \operatorname{supp} \nu\right\}
$$

Then $G_{\nu}^{*}$ and $N_{\nu}^{*}$ are algebraic subgroups of $G^{*}$, and $N_{\nu}^{*}$ is a cocompact, normal subgroup of $G_{\nu}^{*}$.

Corollary 4.3. Suppose $\Gamma$ is a closed subgroup of a Lie group $G$, and $U$ is a unipotent subgroup of $G$. If $\mu$ is an ergodic, $U$-invariant probability measure on $\Gamma \backslash G$, then there is some $g \in G$ such that

(a) the translated measure $\mu g$ is supported on $\Pi N_{G}\left(\Gamma^{\circ}\right)$; 
(b) $\mu g$ is an ergodic, invariant measure for the unipotent subgroup $g^{-1} U g$; and

(c) $g^{-1} U g$ is a subgroup of $N_{G}\left(\Gamma^{\circ}\right)$.

Proof. Let $G^{*}$ be the Zariski closure of Ad $G$ in the real-algebraic group GL( $\left.\mathscr{G}\right)$ where $\mathscr{G}$ is the Lie algebra of $G$, and let $\Gamma^{*}$ be the Zariski closure of $\operatorname{Ad}_{G} \Gamma$ in $G^{*}$. The $U$-invariant probability measure $\mu$ pushes to an ( $\operatorname{Ad}_{G} U$ )-invariant probability measure $\nu=\left(\operatorname{Ad}_{G}\right)_{*} \mu$ on $\Gamma^{*} \backslash G^{*}$. Define $G_{\nu}^{*}$ and $N_{\nu}^{*}$ as in Theorem 4.2. Then $\operatorname{Ad}_{G} U \subset G_{\nu}^{*}$. Any compact real-algebraic group (such as $\left.G_{\nu}^{*} / N_{\nu}^{*}\right)$ has no algebraically unipotent elements, so we must have $\operatorname{Ad}_{G} U \subset N_{\nu}^{*}$. Therefore $N_{\nu}^{*}$ is ergodic. By definition, $N_{\nu}^{*}$ acts trivially on the support of $\nu$, so ergodicity implies that the support of $\nu$ is a single point $\Gamma^{*} g^{*}$ in $\Gamma^{*} \backslash G^{*}$.

Since $\nu$, being the image of $\mu$, is supported on $\Gamma^{*} \cdot(\operatorname{Ad} G)$, we may assume that $g^{*}=\operatorname{Ad}_{G} g$ for some $g \in G$. If we replace $\mu$ by its translate $\mu \cdot g^{-1}$, then we may assume $g=e$. This means that $\nu$ is the point mass at $\Gamma^{*} e$ in $\Gamma^{*} \backslash G^{*}$.

Therefore $N_{\nu}^{*}=\Gamma^{*}$. Hence $\left(\operatorname{Ad}_{G}\right)^{-1}\left(N_{\nu}^{*}\right) \subset N_{G}\left(\Gamma^{\circ}\right)$ (see Lemma 4.1). Since $\operatorname{Ad}_{G} U \subset N_{\nu}^{*}$, this implies $U \subset N_{G}\left(\Gamma^{\circ}\right)$. Since $\nu$ is supported on $\Gamma^{*} \backslash \Gamma^{*} e$, we see that $\mu$ is supported on $\Gamma \backslash N_{G}\left(\Gamma^{\circ}\right)$.

Lemma 4.4. Let $\Gamma, X, U$ be subgroups of a Lie group $G$. Assume $\Gamma$ is discrete, $X$ is connected, and $U$ is unipotent. If there are only finitely many $U$-translates of the $X$-orbit $\Gamma \Gamma X$ in $\Gamma \mid G$, then $U$ normalizes $X$.

Proof. Since there are only finitely many $U$-translates of $\Gamma \Gamma X$, the elements of $U$ that fix $\Gamma \Gamma X$ must form a subgroup of finite index. By Lemma 4.5, it suffices to show that this subgroup normalizes $X$. Thus, given $g \in G$, it suffices to show that if $\Gamma X g=\Gamma X$, then $g \in N_{G}(X)$. Since $e \in \Gamma X$, and $\Gamma X g=\Gamma X$, we must have $g^{-1} \in \Gamma X$. Thus, we may write $g^{-1}=\gamma x$, for some $\gamma \in \Gamma$ and some $x \in X$. Since $\Gamma$ is discrete and $X$ is connected, the connected component of $\Gamma X$ containing $e$ is just $X$. Similarly, the connected component containing $e$ of

$$
\Gamma X g=(\Gamma \gamma)(x X) g=\Gamma\left(g^{-1} X g\right)
$$

is $g^{-1} X g$. Since $\Gamma X=\Gamma X g$, then $X=g^{-1} X g$, as desired.

Lemma 4.5. Let $X$ and $U$ be subgroups of a Lie group $G$. Assume $X$ is connected and $U$ is unipotent. If $X$ is normalized by a finite-index subgroup of $U$, then $X$ is normalized by $U$.

Proof. Since $X$ is connected, it suffices to show that the Lie algebra of $X$ is $\left(\operatorname{Ad}_{G} U\right)$-invariant. The Zariski closure $N^{*}$ of $\operatorname{Ad}_{G}\left(N_{U}(X)\right)$ in $\operatorname{GL}(\mathscr{G})$ is a subgroup of finite index in the Zariski closure $U^{*}$ of $\operatorname{Ad}_{G}(U)$. Any Zariskiclosed, algebraically unipotent subgroup of a real-algebraic group is connected $[14$, p. 10$]$, so $U^{*}$ is connected. Hence, $U^{*}$ has no proper subgroups of finite index; therefore $N^{*}=U^{*}$. Hence, the Lie algebra of $X$ is invariant under $U^{*}=N^{*}$, because the stabilizer of any subspace is a Zariski-closed subgroup $[14$, p. 8].

Lemma 4.6 [14, Theorem 2.18, p. 40]. Let $X$ be a finitely-generated, torsionfree, nilpotent group. Then $X$ is isomorphic to a lattice subgroup of some connected, nilpotent Lie group. 
Lemma 4.7. Let a Lie group $G$ act on a Borel space $S$ and let $\mu$ be a probability measure on $S$. Assume $\mu$ is invariant and ergodic, with respect to some unipotent subgroup $U$ of $G$. Suppose $G^{\prime}$ is a Lie subgroup of finite index in $G$. If some ergodic component of $\mu$, with respect to $U \cap G^{\prime}$, is homogeneous for a subgroup of $G^{\prime}$, then $\mu$ is homogeneous for a subgroup of $G$.

Proof. Because $U \cap G^{\prime}$ is a finite-index subgroup of $U$, we know that $U \cap G^{\prime}$ contains a finite-index, normal subgroup $N$ of $U$. Now the finite group $U / N$ acts ergodically on the space of ergodic components of $\mu$, w.r.t. $N$. This means that $\mu$ has only finitely many ergodic components w.r.t. $N$, and $U$ is transitive on the set of these components. Since $N \subset U \cap G^{\prime}$, then $\mu$ has only finitely many ergodic components w.r.t. $U \cap G^{\prime}$. By assumption, some ergodic component $\rho$ is homogeneous for a subgroup of $G^{\prime}$. This means that $\rho$ is supported on a single $X$-orbit, where $X=\operatorname{Stab}_{G^{\prime}}(\rho)$. The definition of $\rho$ implies that $U \cap G^{\prime} \subset X$, so $U \cap G^{\prime}$ normalizes $X^{\circ}$. Then Lemma 4.5 asserts that $U$ normalizes $X^{\circ}$.

Because $X / X^{\circ}$ is countable, some $X^{\circ}$-orbit must have nonzero measure. By ergodicity, $\mu$ is supported on the union of the $U$-translates of this $X^{\circ}$ orbit. Since $U$ normalizes $X^{\circ}$, any $U$-translate of the $X^{\circ}$-invariant measure on an $X^{\circ}$-orbit is the $X^{\circ}$-invariant measure on another $X^{\circ}$-orbit. Since any two distinct $X^{\circ}$-orbits are disjoint, and $\mu$ is a probability measure, this implies that $\mu$ is the sum of $X^{\circ}$-invariant measures on finitely many $X^{\circ}$-orbits. Then $\mu$ is $X^{\circ} U$-invariant, and supported on a single $X^{\circ} U$-orbit.

The following lemmas are probably known, but convenient references do not seem to be available.

Lemma 4.8. Let $U$ be a closed, unipotent subgroup of a connected Lie group. Suppose $U$ acts ergodically on a probability space $S$. Then $U$ has a finitelygenerated, nilpotent subgroup $X$ that is ergodic on $S$.

Proof. Any unipotent subgroup of a connected Lie group is nilpotent (cf. Engel's Theorem $\left[6, \S \mathrm{V} .2\right.$, pp. 63-67]), so $U$ is nilpotent. Let $S_{0}$ be (almost) any ergodic component of the action of $U^{\circ}$ on $S$. Since $U^{\circ}$ is connected and nilpotent, some element $u$ of $U^{\circ}$ is ergodic on $S_{0}$ [21, Proposition 5.1].

Since $U / U^{\circ}$ is finitely generated (Lemma 4.9), we may choose a finite set $F$ of elements of $U$ that generates $U / U^{\circ}$. Then the subgroup generated by $F U$ $\{u\}$ is ergodic on $S$.

Definition [1, Definition 0.1]. A connected Lie group $G$ is almost algebraic if $G$ is of the form $G=N \times(L \times T)$, where $N$ is the nilradical of $G, L$ is a closed, semisimple subgroup, and $T$ is a torus (in other words, $T$ is a closed, abelian subgroup such that $\operatorname{Ad}_{G} t$ is semisimple, for every $t \in T$ ).

Lemma 4.9. Let $U$ be a closed, unipotent subgroup of a connected Lie group $G$. Then $U / U^{\circ}$ is finitely generated.

Proof. By replacing $G$ with its universal cover, we may assume $G$ is simply connected. Then, by replacing $G$ with a larger group, we may assume $G$ is almost algebraic [1, Theorem 0.1 ], so we may write $G=N \rtimes(L \times T)$, where $N$ is the nilradical of $G, L$ is a closed, semisimple subgroup, and $T$ is a torus. There are natural homomorphisms $\pi_{L}: G \rightarrow L$ and $\pi_{T}: G \rightarrow T$.

The elements of $\pi_{T}(U)$ are unipotent, because elements of $U$ are unipotent, but the elements of $\pi_{T}(U)$ are also semisimple, because elements of $T$ are 
semisimple. Hence $\pi_{T}(U) \subset Z(G)$, which means $U \subset N L \cdot(T \cap Z(G))$. Now $T \cap Z(G)$ is discrete, because $T \cap N=e$, so we must have $U^{\circ} \subset N L$.

The subgroup $\pi_{L}(U)$ is a unipotent subgroup of $L$, hence contained in a maximal unipotent subgroup $V$ of $L$. Then $U \cap N L \subset N V$. The maximality of $V$ implies that $\operatorname{Ad}_{L}(V)$ is a maximal algebraically unipotent subgroup of the real-algebraic group $\operatorname{Ad} L$. This implies that $\operatorname{Ad}_{L}(V)$ is a connected, simplyconnected, nilpotent group (see [24, Theorem 3.6.3, p. 197]). Since $Z(L)$ is discrete, then $V$ is of the form $V=Z(L) \times V^{\circ}$, and $V^{\circ}$ is a connected, simplyconnected, nilpotent Lie group. We have $(N V)^{\circ}=N V^{\circ}$, so $U^{\circ} \subset N V^{\circ}$.

We have the following increasing chain of normal subgroups from $U^{\circ}$ to $U$ :

$$
U^{\circ} \triangleleft U \cap N V^{\circ} \triangleleft U \cap N V=U \cap N L \triangleleft U \cap[N L \cdot(T \cap Z(G))]=U .
$$

By verifying that each of the three nontrivial quotients in this chain is finitely generated, we conclude that the composite quotient $U / U^{\circ}$ is finitely generated, as desired.

(1) Because $U^{\circ} \subset N V^{\circ}$, we have $\left(U \cap N V^{\circ}\right)^{\circ}=U^{\circ}$. Since $U \cap N V^{\circ}$ is a closed subgroup of the connected nilpotent Lie group $N V^{\circ}$, we know that $\left(U \cap N V^{\circ}\right) /\left(U \cap N V^{\circ}\right)^{\circ}$ is finitely generated [14, Proposition 3.8, p. 53].

(2) The second quotient, $(U \cap N V) /\left(U \cap N V^{\circ}\right)$, is isomorphic to a subgroup of $N V / N V^{\circ} \cong V / V^{\circ} \cong Z(L)$. The fundamental group of any connected Lie group is a finitely-generated, abelian group (because any connected Lie group is homeomorphic to the cartesian product of a compact group and a Euclidean space $\mathbf{R}^{n}[14, \S P .1 .8]$, and the fundamental group of any compact group is a finitely-generated, abelian group [2, Corollary 1.4 .3$, p. 5]). Thus $Z(L)$, the fundamental group of $L / Z(L)$, is a finitely-generated, abelian group. Any subgroup of a finitely generated, abelian group is finitely generated.

(3) The final nontrivial quotient is isomorphic to a subgroup of $T \cap Z(G)$. Because $T \cap Z(G)$ is a discrete subgroup of the connected, abelian group $T$, we know that $T \cap Z(G)$ is a finitely-generated abelian group [14, Corollary 2 of Theorem 2.10, p. 33]. Subgroups of finitely generated abelian groups are finitely-generated [14, Theorem 2.7 , p. 31].

\section{ACTIONS OF SEMISIMPLE GROUPS OR LATTICES}

Corollary 5.1. Suppose $G$ is a connected, semisimple Lie group, and $\Gamma \backslash G$ is a faithful, finite-volume homogeneous space of $G$. Let $U$ be any unipotent subgroup of $G$ that acts ergodically on $\Gamma \mid G$. Then any quotient of the $U$-action on $\Gamma \mid G$ is of the form $\left(N \Gamma^{\prime}\right) \backslash G / K$, where

(1a) $N$ is a closed, normal subgroup of $G$ such that $N \Gamma$ is closed;

(1b) $\Gamma$ is a lattice containing $\Gamma$; and

(2) $K$ is a compact group, consisting of affine maps on $\left(N \Gamma^{\prime}\right) \backslash G$ that centralize $U$.

Proof. Suppose $\Lambda \backslash G / K$ is a central double-coset quotient of $\Gamma \backslash G$. Now $K$ contains a subgroup $K^{\prime}$ of finite index that consists entirely of translations (see Lemma 5.3). Let

$$
X=\left\{x \in G \mid \Lambda g x K^{\prime}=\Lambda g K^{\prime} \text { for a.e. } g \in G\right\} .
$$


The Borel Density Theorem implies that $G$ has finite center (see Lemma 5.4). The Moore Ergodicity Theorem implies that $X$ contains a closed, normal subgroup $N_{1}$ of $G$ such that $X / N_{1}$ is compact (see Lemma 5.2). Then, because $G$ is semisimple with finite center and $N_{1}$ is a normal subgroup, we may write $G=N_{1} H$, where $H$ is a normal subgroup of $G$, and $N_{1} \cap H$ is finite. Note that $X=N_{1} \cdot(X \cap H)$, and $X \cap H$ is compact. By definition, $X \supset K^{\prime}$. By replacing $\Lambda$ with $\Lambda N_{1}$, we may assume $K^{\prime}=H \cap X$, which means $K^{\prime}$ is compact. Then $K$ is also compact.

Let $N$ be the identity component of $\Lambda$. The Borel Density Theorem implies that $N$ is a normal subgroup of $G$, that $\Gamma$ is discrete, and that $G$ has finite center (see Lemma 5.4). We may write $G=N H$, where $H$ is a closed, normal subgroup of $G$, and $N \cap H$ is finite. Note that $\Lambda=N \cdot(H \cap \Lambda)$. Let $\Gamma^{\prime}=$ $\Gamma \cdot(H \cap \Lambda)$, so $\Lambda=N \Gamma^{\prime}$. Then $\Gamma^{\prime}$ obviously contains $\Gamma$, and $\Gamma^{\prime}$ is discrete because its projections into both $N$ and $H$ are discrete.

Lemma 5.2. Let $\Gamma$ be a lattice in a connected, semisimple Lie group $G$, and assume $Z(G)$ is finite. Suppose $\psi$ is a measurable function on $\Gamma \backslash G$, and let

$$
X=\{x \in G \mid \psi(s x)=\psi(s) \text { for a.e. } s \in \Gamma \backslash G\} .
$$

Then there is a closed, normal subgroup $N$ of $G$, contained in $X$, such that $X / N$ is compact.

Proof. This follows from the Moore Ergodicity Theorem [8, Theorem 2] as in the proof of [8, Theorem 5].

Lemma 5.3. Let $\Lambda$ be a lattice in a connected, semisimple Lie group $G$, and assume some unipotent subgroup of $G$ acts ergodically on $\Lambda \backslash G$. Then the translations form a subgroup of finite index in $\mathrm{Aff}(\Lambda \backslash G)$.

Proof. Since $G$ is semisimple, the group $\operatorname{Inn}(G)$ of inner automorphisms of $G$ has finite index in the full automorphism group $\operatorname{Aut}(G)$ of $G$ [7, Theorem 27.4(b), pp. 166-167]. Hence, $N_{\operatorname{Inn}(G)}(\Lambda)$ is a subgroup of finite index in $N_{\text {Aut }(G)}(\Lambda)$.

We know that $\Lambda$ is of finite index in $N_{G}(\Lambda)$ (cf. Lemma 5.4). This means that the inner automorphisms arising from elements of $\Lambda$ form a subgroup of finite index in $N_{\operatorname{Inn}(G)}(\Lambda)$, and hence a subgroup of finite index in $N_{\operatorname{Aut}(G)}(\Lambda)$. Any affine map whose automorphism component is the conjugation by some element of $\Lambda$ is a translation: if $\varphi(\Lambda s)=\Lambda s^{\lambda} g$, then $\varphi(\Lambda s)=\Lambda\left(\lambda^{-1} s \lambda\right) g=$ $\Lambda s \cdot(\lambda g)$.

Lemma 5.4. Suppose $G$ is a connected, semisimple Lie group, and $\Gamma \backslash G$ is a finite-volume homogeneous space of $G$. Assume some unipotent subgroup $U$ of $G$ acts ergodically on $\Gamma G$. Then $\Gamma^{\circ} \triangleleft G$. If $\Gamma \backslash G$ is faithful, then $\Gamma$ is discrete, $Z(G)$ is finite, and $\Gamma$ is of finite index in $N_{G}(\Gamma)$.

Proof. Dani's version of the Borel Density Theorem implies that $\Gamma^{\circ} \triangleleft G$ (see Corollary 4.3).

If $\Gamma \backslash G$ is faithful, then $\Gamma$ cannot contain any normal subgroup of $G$, so $\Gamma^{\circ}=e$, which means $\Gamma$ is discrete. This implies that $N_{G}(\Gamma)^{\circ} \subset C_{G}(\Gamma)^{\circ}$. Now $\Gamma$ centralizes the Lie algebra of $C_{G}(\Gamma)$, and, from the proof of Corollary 4.3, we know that the Zariski closure $\Gamma^{*}$ of $\Gamma$ in GL( $\left.\mathscr{G}\right)$ contains all of $\operatorname{Ad} G$, so we know that Ad $G$ centralizes the Lie algebra of $C_{G}(\Gamma)$. Hence $G$ centralizes $C_{G}(\Gamma)^{\circ}$; in other words, $C_{G}(\Gamma)^{\circ} \subset Z(G)$. Because $Z(G)$ is discrete (since 
$G$ is semisimple) and $C_{G}(\Gamma)^{\circ}$ is connected, we conclude that $C_{G}(\Gamma)^{\circ}=e$. Hence $N_{G}(\Gamma)^{\circ}=e$, so $N_{G}(\Gamma)$ is discrete. This means that $N_{G}(\Gamma)$ is a lattice containing $\Gamma$. Hence $\Gamma$ is of finite index in $N_{G}(\Gamma)$ [14, Lemma 1.6].

Since $\Gamma$ does not contain any normal subgroup of $G$, we must have $\Gamma \cap$ $Z(G)=e$. Since $\Gamma \cdot Z(G) \subset N_{G}(\Gamma)$, and $\Gamma$ has finite index in $N_{G}(\Gamma)$, this implies that $Z(G)$ is finite.

Corollary 5.5. Let $G$ be a connected, semisimple Lie group with no compact factors. Immerse $G$ in some connected group $H$, and let $\Lambda$ be a closed subgroup of $H$. Then every ergodic G-invariant probability measure on $\Lambda \backslash H$ is homogeneous for a subgroup of $H$.

Proof. Let $U$ be a maximal unipotent subgroup of $G$. The Mautner Phenomenon (Theorem 5.7) implies that any ergodic $G$-action on a probability space remains ergodic when it is restricted to $U$. Thus, Theorem 1.2 implies that any ergodic $G$-invariant probability measure on $\Lambda \backslash H$ is homogeneous.

Corollary 5.6. Let $G$ be a connected, semisimple Lie group with no compact factors. Immerse $G$ in some connected group $H$, and let $\Lambda \backslash H$ be a finitevolume homogeneous space of $H$, on which $G$ acts ergodically. Then every measurable quotient of the $G$-action on $\Lambda \backslash H$ is a central double-coset quotient.

Proof. Corollary 5.5 implies that any ergodic invariant probability measure for the diagonal $G$-action on $(\Lambda \backslash H) \times(\Lambda \backslash H)$ is homogeneous for a subgroup of $H \times H$. Hence Theorem 2.1 asserts that every quotient of the $G$-action on $\Lambda \backslash H$ is a central double-coset quotient.

Theorem 5.7 [9, Theorem 1.1]. Let a connected Lie group $G$ act on a probability space $S$, and let $U$ be a connected subgroup of $G$. Then there is a closed, normal subgroup of $G$ such that the closure of the image of $U$ in $\operatorname{Ad}(G / N)$ is precompact, and such that every $U$-invariant measurable function on $S$ is essentially $\mathrm{N}$-invariant.

Corollary 5.8. Let $\Gamma$ be a lattice in a connected, semisimple Lie group $G$ with no compact factors. Suppose $\Gamma$ acts automorphically on $\Lambda \backslash N$. Then any ergodic $\Gamma$-invariant probability measure $\mu$ on $\Lambda \backslash N$ is homogeneous for a subgroup of the semidirect product $\Gamma \propto N$.

Proof. Let $G^{\prime}=G \propto N$ and $\Gamma^{\prime}=\Gamma \times \Lambda \subset G^{\prime}$. There is a natural embedding of $\Lambda \backslash N$ in $\Gamma^{\prime} \backslash G^{\prime}$, so we may think of $\mu$ as a $\Gamma$-invariant probability measure on $\Gamma^{\prime} \backslash G^{\prime}$ that is supported on $\Gamma^{\prime} \backslash \Gamma^{\prime} N$. By averaging this measure over a $G$ orbit in the space of probability measures, we obtain a $G$-invariant probability measure $\mu^{\prime}$. Corollary 5.5 asserts that there is a subgroup $X$ of $G^{\prime}$ such that $\mu^{\prime}$ is the $X$-invariant probability measure on some $X$-orbit $s X$. Replacing $s$ by a $G$-translate, we may assume $s \in N$. Then, replacing $\mu$ and $s$ by $N$-translates, we may assume $s=e$.

Since $\mu^{\prime}$ is $G$-invariant, we may assume $G \subset X$ (by replacing $X$ with the full stabilizer of $\left.\mu^{\prime}\right)$. Let $M=X \cap N$; then $X=G \ltimes M$. Now $\Gamma \backslash G \cong\left(\Gamma^{\prime} N\right) \backslash G^{\prime}$ is naturally a quotient of $\Gamma^{\prime} \backslash G^{\prime}$. The fibers of this quotient are simply the $N$ orbits on $\Gamma^{\prime} \backslash G^{\prime}$, so we can decompose $\mu^{\prime}$ essentially uniquely into probability measures supported on the $N$-orbits [22, $\S 3, \mathrm{pp}$. 26-33]. The construction of $\mu^{\prime}$ provides an explicit way to do this. Namely, for $g \in G$, the probability measure supported on $\Gamma^{\prime} \backslash \Gamma^{\prime} g N$ is simply the translate $\mu \cdot g$ of $\mu$. 
We can also construct the decomposition of $\mu^{\prime}$ by looking at the subgroup $X$. Since $\left(\Gamma^{\prime} \cap X\right) \backslash X \cong \Gamma^{\prime} \backslash \Gamma^{\prime} X$ has a finite $X$-invariant measure, and $\Gamma^{\prime} \cap X \subset$ $\Gamma M$, we know that $(\Lambda \cap M) \backslash M \cong\left(\Gamma^{\prime} \cap X\right) \backslash \Gamma M$ supports a $\Gamma M$-invariant probability measure $\rho$, and the $X$-invariant probability measure on $\left(\Gamma^{\prime} \cap X\right) \backslash X$ decomposes into the translates of $\rho[14$, proof of Lemma 1.6, p. 20]. By identifying $\left(\Gamma^{\prime} \cap X\right) \backslash X$ with the orbit $s X$, this provides a decomposition of $\mu^{\prime}$. By uniqueness of the decomposition, then we conclude that almost every $G$ translate of $\mu$ is a $G$-translate of $\rho$. It follows that every translate, including $\mu$ itself, is a $G$-translate of $\rho$. Since the only $G$-translate of $\rho$ that is supported on $\Lambda \backslash N$ is $\rho$ itself, we conclude that $\mu=\rho$. By the definition of $\rho$, then it is clear that $\mu$ is homogeneous for a subgroup of $\Gamma M$.

Example 5.9. The arithmetic group $\Gamma=\mathrm{SL}_{n}(Z)$ is a lattice in the (almost) simple group $G=\mathrm{SL}_{n}(\mathbf{R})$. The natural action of $G$ on $\mathbf{R}^{n}$ gives rise to an action of $\Gamma$ on $T^{n}=\Lambda \backslash N$, where $N=\mathbf{R}^{n}$ and $\Lambda=\mathbb{Z}^{n}$. Assume $n \geq 2$, so that the $\Gamma$-action is ergodic. Corollary 1.4 asserts that every quotient of this action is of the form $\Lambda^{\prime} \backslash N / K$, where

(1) $\Lambda^{\prime}$ is a closed, $\Gamma$-invariant subgroup of $N$ containing $\Lambda$; and

(2) $K$ is a closed group of affine maps on $\Lambda^{\prime} \backslash N$ such that

(a) after discarding a set of measure 0 , the orbit space $\Lambda^{\prime} \backslash N / K$ is countably separated; and

(b) every affine map in $K$ commutes with every element of $\Gamma$.

There are no nontrivial, connected, closed, $\Gamma$-invariant subgroups of $\mathbf{T}^{n}$, so $\Lambda^{\prime}$ must be discrete. This means that $\Lambda^{\prime} \backslash \mathbf{R}^{n}$ is an $n$-torus finitely covered by $\mathbf{T}^{n}$.

We claim that the only nontrivial element of $K$ (if there is one) is the involution $\sigma$ defined by $\sigma(x)=-x$. Note that 0 is the only fixed point of the $\Gamma$-action on $\Lambda^{\prime} \backslash N$. Since $K$ centralizes $\Gamma$, this implies that every element of $K$ fixes 0 . An affine map that fixes the identity is an automorphism, so every element of $K$ is an automorphism. The claim now follows from the fact that the centralizer of $\mathrm{SL}_{n}(\mathbb{Z})$ in $\operatorname{Aut}\left(\Lambda^{\prime} \backslash N\right) \cong \mathrm{GL}_{n}(\mathbb{Z})$ is the subgroup generated by the involution $\sigma$.

The claim of the preceding paragraph means that the quotient $\Lambda^{\prime} \backslash N / K$ is either $\Lambda^{\prime} \backslash N$ (a torus) or $\Lambda^{\prime} \backslash N /\langle\sigma\rangle$ (an orbifold; see [23, §2] for an introduction to orbifolds). For $n=2$, the orbifold $\Lambda^{\prime} \backslash N /\langle\sigma\rangle$ is homeomorphic to the 2 sphere, but for $n=3$, the orbifold is not a manifold (it has eight branch points, and a neighborhood of any of these points is a cone on a projective plane). I thank Colin Adams for a helpful discussion that clarified these matters for me.

\section{REFERENCES}

1. L. Auslander and J. Brezin, Almost algebraic Lie algebras, J. Algebra 8 (1968), 295-313.

2. N. Bourbaki, Groupes et algèbres de Lie, Chapitre 9: Groupes de Lie réels compacts, Masson, Paris, 1982.

3. J. Brezin and C. C. Moore, Flows on homogeneous spaces: a new look, Amer. J. Math. 103 (1981), 571-613.

4. S. G. Dani, On ergodic quasi-invariant measures of group automorphism, Israel J. Math. 43 (1982), 62-74.

5. A. del Junco and D. Rudolph, On ergodic actions whose self-joinings are graphs, Ergodic Theory Dynamical Systems 7 (1987), 531-557. 
6. G. P. Hochschild, Basic theory of algebraic groups and Lie algebras, Springer-Verlag, New York, 1981.

7. J. E. Humphreys, Linear algebraic groups, Springer-Verlag, New York, 1975.

8. C. C. Moore, Ergodicity of flows on homogeneous spaces, Amer. J. Math. 88 (1966), 154-178.

9. 155-169.

10. G. D. Mostow, On the fundamental group of a homogeneous space, Ann. of Math. 66 (1957), 249-255.

11. K. Park, GL(2, Z) action on $T^{2}$, (preprint).

12. Metric classification of ergodic nilflows and unipotent affines, Amer. J. Math. 93 (1971), 819-828.

13. W. Parry, Dynamical representations in nilmanifolds, Compositio Math. 26 (1973), 150-174.

14. M. S. Raghunathan, Discrete subgroups of Lie groups, Springer, New York, 1972.

15. M. Ratner, Rigidity of horocycle flows, Ann. of Math. 115 (1982), 597-614.

16. __ Factors of horocycle flows, Engodic Theory Dynamical Systems 2 (1982), 465-489.

17. __ Horocycle flows: joinings and rigidity of products, Ann. of Math. 118 (1983), 277-313.

18. __ Ergodic theory in hyperbolic space, Contemp. Math. 26 (1984), 309-334.

19. __ Strict measure rigidity for unipotent subgroups of solvable groups, Invent. Math. 101 (1990), 449-482.

20. $\ldots$, On measure rigidity of unipotent subgroups of semisimple groups, Acta Math. 165 (1990), 229-309.

21. _, On Raghunathan's measure conjecture, Ann. of Math. 134 (1991), 545-607.

22. V. A. Rohlin, On the fundamental ideas of measure theory, Amer. Math. Soc. Transl. (1) 10 (1962), 1-54; English transl. of Mat. Sb. (N.S.) 25(67) (1949), 107-150.

23. P. Scott, The geometries of 3-manifolds, Bull. London Math. Soc. 15 (1983), 401-487.

24. V. S. Varadarajan, Lie groups, Lie algebras, and their representations, Springer, New York, 1984.

25. D. Witte, Zero-entropy affine maps on homogeneous spaces, Amer. J. Math. 109 (1987), 927961.

26. R. J. Zimmer, Ergodic theory and semisimple groups, Birkhäuser, Boston, Mass., 1984.

Department of Mathematics, Arizona State University, Tempe, Artzona 85287

Current address: Department of Mathematics, Williams College, Williamstown, Massachusetts 01267

E-mail address: dritteorilliams.edu 